\title{
Macroelectrode Responses with Stimulation and Recording within Hippocampus and Related Structures during Different States of Consciousness
}

\author{
JAMES B. RANCK, JR. ${ }^{1}$ \\ Department of Physiology, The University of Michigan, Ann Arbor, Michigan 48104
}

Received September 25, 1968

\begin{abstract}
Three or four pairs of bipolar electrodes were chronically implanted in the hippocampal formation, subicular area, and entorhinal cortex of rats, all on the same side. The EEG, EOG, and neck EMG were also recorded. Responses due to electrical stimulation of one electrode pair were recorded in the other electrode pairs during slow-wave sleep, paradoxical sleep, and arousal. The stimuli were single, constant current pulses once every $5 \mathrm{sec}$. Most responses did not change with different states of consciousness. All responses which were smaller during paradoxical sleep than during slow-wave sleep were also small during arousal. Most changes were in this category. All of the responses which were larger during paradoxical sleep than in slow-wave sleep were the same in arousal and slow-wave sleep. Responses in hippocampus or fascia dentata from stimulation of other parts of these structures often changed, usually decreasing during paradoxical sleep and arousal. Responses in subiculum, presubiculum, or entorhinal cortex from stimulation of hippocampus or fascia dentata usually changed, usually decreasing with paradoxical sleep and arousal. Responses in entorhinal cortex or presubiculum from stimulation of subiculum increased in paradoxical sleep. Responses in fornix and $\mathrm{CA}_{3}$ from stimulation of presubiculum decreased in paradoxical sleep. An important negative finding was that no responses due to stimulation of entorhinal cortex changed in different states of consciousness. No clear correlation of the changes with phasic phenomena of paradoxical sleep was seen.
\end{abstract}

\section{Introduction}

The activity in the hippocampal formation changes in different states of consciousness. During paradoxical sleep it develops a theta rhythm (2), there are marked impedance increases in the subiculum and presubiculum (4), blood flow more than doubles (5), and unit activity decreases (3). There is also theta activity in hippocampus in some forms of arousal. In this study, macroelectrode responses were recorded in hippocampus and related structures due to electrical stimulation of these same structures dur-

1 This study was supported by grant NIH NB-04352 from The National Institutes of Health. Miss Christine McBride, Mrs. Ann Maxwell, and Drs. Leroy Vernon and Alan Fertziger aided at various stages. 
ing different states of consciousness. Not all responses changed with change in state of consciousness, but those which did showed a pattern.

\section{Methods}

Sprague-Dawley male rats weighing $250-500 \mathrm{~g}$ had electrodes stereotaxically implanted. There were two EEG electrodes (recording from stainless-steel screws in or on neocortex), two neck-muscle EMG electrodes (.006-inch Nichrome wires), and three or four pairs of brain electrodes (two twisted .007-inch diameter stainless-steel wires, insulated except at the blunt tips). Four animals had two electro-occulogram (EOG) electrodes (.006-inch diameter Nichrome wires subcutaneous and lateral to each orbit). Recording sessions occurred $7-40$ days after surgery. The rats were run in $24-\mathrm{cm}$ diameter glass animal jars. The night before recording sessions they were deprived of paradoxical sleep by the inverted flowerpot-in-water method. Often the rat was run for many days consecutively, in which case it would be deprived for many consecutive nights, but allowed to sleep during the day. There was no overt response to most stimuli at the intensities used, but a few did show contraction of the scrotum. All the rats fell asleep as readily as unstimulated rats, so it seems unlikely that the stimuli caused any significant pain.

Continuous records were taken of EEG, EOG, and nuchal EMG on an ink polygraph at a paper speed of $1 \mathrm{~mm} / \mathrm{sec}$. Electrical stimuli were given through one pair of brain electrodes and the responses recorded on an oscilloscope from two other pairs. Recording was usually bipolar from the two electrodes of the pair, but some recordings were taken between one depth electrode and one EEG electrode, after showing that no responses were present in the EEG electrodes.

The stimuli were single constant-current pulses, once every $5 \mathrm{sec}$. Responses were recorded at slightly above threshold, and usually at two or three other steps up to a maxinum current of $1 \mathrm{mamp}, 1 \mathrm{msec}$, or more commonly, at a maximum response which occurred with stimuli less than 1 mamp, $1 \mathrm{msec}$. Most thresholds were about $0.3 \mathrm{msec}$ and $0.1-0.5 \mathrm{mamp}$. All possible combinations of stimulating and recording were used. All electrodes were in dorsal hippocampal formation, dorsal, or posterior subiculum or presubiculum, or entorhinal cortex, and all were on the same side.

In thirteen rats, during the early part of the study, responses were photographed during slow-wave sleep, paradoxical sleep and arousal, but usually none of these episodes was repeated at the same stimulus strength. Later, in seven rats an episode of slow-wave sleep (or in a few cases. paradoxical sleep) was repeated, to be sure that the responses were stable. (We will call these "well-run" rats.) In almost all cases the responses were indeed stable, 
but a few changes were seen and the data were rejected. The entire series of responses at a single-current strength was recorded within $20 \mathrm{~min}$. At least ten individual responses were recorded in each episode.

No attempt was made to distinguish episodes of spindling during slowwave sleep. The slow-wave sleep episodes were ones with the largest amplitude EEG. Arousal was defined as low-voltage activity on the EEG and attentive behavior. In most cases this was elicited by hitting the jar or by snapping fingers. Clearly, this is a very crude procedure which includes an uncontrolled amount of fear. However, the changes with "arousal" were always consistent. In almost all cases, the rat was not moving during the recordings of arousal. No search for hippocampal theta rhythms was made.

At the end of the run, a rat was perfused with formalin and frozen sections were cut and stained with cresyl violet for identification of electrode location.

The data were read by eye directly from the film; averaging methods were not used. Therefore, the possibility of changes occurring during the phasic episodes of paradoxical sleep could be sought and investigated. The responses were all fairly regular so that changes of $20 \%$ or less could be readily detected.

\section{Results}

Most responses did not change in different states of consciousness. Thirty-three of the 53 responses in the well-run rats did not change, and in the less well-run group, 23 of 44 did not change. Those responses which did show changes usually did not show changes at all current strengths. However, in the well-run rats all those responses which changed did so at more than one current strength.

Figure 1 summarizes the results from the seven well-run rats. Slow-wave sleep is taken as a baseline, and changes in paradoxical sleep are shown. In all cases where there was a decrease in the size of the response during paradoxical sleep, the response also decreased in amplitude during arousal, and sometimes even during slow-wave sleep in which the amplitude of the EEG waves was less than maximum (Fig. 2). In most, however, the response did not decrease unless there was a full and clear arousal. The size of the decreased response with arousal was always about the same as the response during paradoxical sleep. In some cases there was a decrease with paradoxical sleep, but not with arousal at one current strength or vice versa. However, when this occurred, it was found that both would change at some other current strength. There were no responses that changed with arousal which did not decrease with paradoxical sleep at some strength. In all cases where there was an increase in the response during paradoxical sleep, there was no change with arousal (Fig. 3). 


\begin{tabular}{|c|c|c|c|c|c|c|c|c|c|}
\hline & Fornix & $\mathrm{CA}_{3}$ & $\begin{array}{l}\left(C A_{3}, f, d\right. \\
\left.\text { and } C A_{4}\right)\end{array}$ & $C A_{1}$ & f.d. $+\mathrm{CA}_{4}$ & Subic. & Ento. & Presubic. & $\begin{array}{l}\text { Subic. } \\
\text { and } C A_{1}\end{array}$ \\
\hline \multicolumn{10}{|l|}{ Fornix } \\
\hline \multicolumn{10}{|l|}{$\mathrm{CA}_{3}$} \\
\hline $\begin{array}{l}\left(\mathrm{CA}_{3}, \mathrm{f} . \mathrm{d} .\right. \\
\left.\text { and } \mathrm{CA} \mathrm{A}_{4}\right)\end{array}$ & & & $\begin{array}{lll}0 & 0 \\
0 & 0 & 0\end{array}$ & & & & $-\infty$ & & \\
\hline \multicolumn{10}{|l|}{$C A_{1}$} \\
\hline \multicolumn{10}{|l|}{ f.d. $+\mathrm{CA}_{4}$} \\
\hline Subiculum & & $?$ & & $\begin{array}{l}1 \\
0 \\
0 \\
1\end{array}$ & & & $\begin{array}{l}0 \\
0\end{array}$ & & o \\
\hline Entorhinal & & 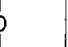 & 0000 & 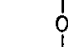 & 0 & 00 & & & \\
\hline \multicolumn{10}{|l|}{ Presubic. } \\
\hline $\begin{array}{l}\text { Subiculum } \\
\text { and } C A_{I}\end{array}$ & & & & $\begin{array}{l}1 \\
0\end{array}$ & b & 0 & & & \\
\hline
\end{tabular}

FIG. 1. The list of structures on the left indicates the location of the stimulating electrode tip. The list of structures on the top indicates the location of the recording electrode tip. Slow-wave sleep is the baseline. The changes are changes during paradoxical sleep. Symbols on a line indicate that the electrode tip was on the border of these structures. Abbreviation: f.d. = fascia dentata. Parasubiculum is included in the presubiculum category as I cannot distinguish them in Nissl-stained sections. Zero $(0)=$ no change in paradoxical sleep; minus sign $(-)=$ a decrease in response with paradoxical sleep; plus sign $(+)=$ an increase in response with paradoxical sleep. The relatively heavier + and - signs indicate relatively greater increases or decreases. If no response could be elicited, which happened in a few cases, there is no notation on the figure.

The major pattern from Fig. 1 is a negative one. There are no changes in responses due to stimulation of entorhinal cortex. There are many changes in hippocampus due to stimulation within hippocampus, and these changes are usually decreases. There are many changes of response in subiculum, presubiculum, and entorhinal cortex due to stimulation of hippocampus, and these changes are usually decreases. There are decreases of response in $\mathrm{CA}_{3}$ or fornix, or both, due to stimulation of presubiculum. There usually are no changes in response due to stimulation of subiculum, and those changes which do occur are increases. There were no direct electrode hits in CA1, so it is not clear how this part enters into the pattern. No contralateral relations were studied. In the 14 rats which were less well studied, this same pattern was apparent, and stimulation of entorhinal cortex, in particular, did not lead to significant changes in response in other parts of the hippocampal formation in ten more cases. The changes occurring with paradoxical sleep were fairly constant throughout the whole 


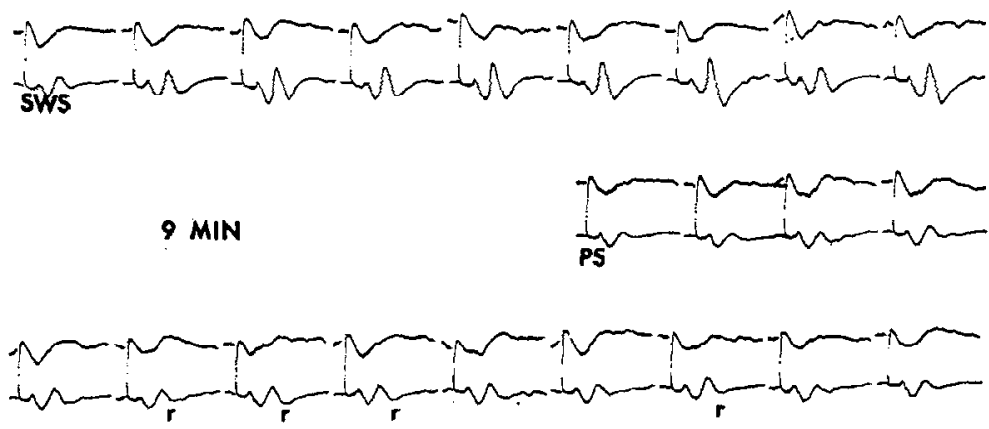

3 MIN
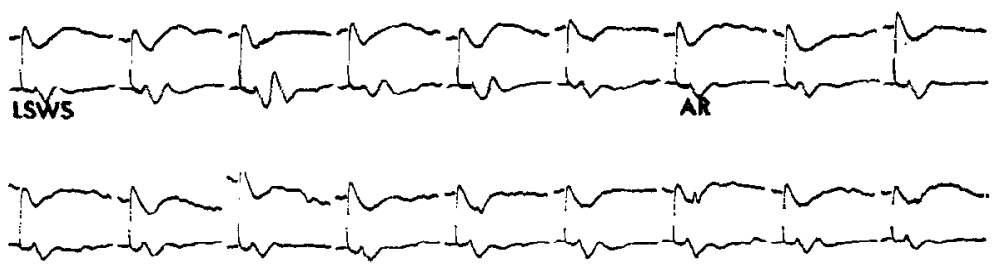

$\longrightarrow I$

FIG. 2. Stimulating electrode in fascia dentata, $\mathrm{CA}$, and $\mathrm{C} \Lambda_{3}$, top recording from $\mathrm{CA}_{4}$ and $\mathrm{CA}_{3}$ (bipolar), bottom recording from entorhinal cortex (bipolar). A response is elicited every $5 \mathrm{sec}$ and photographed. All responses are shown except for the intervals noted. Stimulation $0.24 \mathrm{mamp}, 0.3 \mathrm{msec}$. Calibrations $50 \mathrm{msec} ; 200 \mu \mathrm{v}$ top trace, $400 \mu \mathrm{v}$ bottom trace. Abbreviations: $\mathrm{r}=$ during an episode of rapid eye movenent; SWS = slow-wave sleep; PS = paradoxical sleep; LSWS = comparatively low-voitage slow-wave sleep (see text); $A R=$ arousal. The response on the top trace does not change. The response on the bottom trace decreases with PS, LSWS, and AR.

episode (Figs. 2 and 3 ). In the four rats with EOG recordings, correlation of response with the phasic episodes was looked for. No clear correlation was found.

Strength duration curves were taken on 12 responses, representing a sampling of all locations. Chronaxies varied from 0.24 to $0.46 \mathrm{msec}$, and most were $0.3 \mathrm{msec}$.

\section{Discussion}

Major changes in blood flow to the brain occur during paradoxical sleep (5), so if there is any change in response during paradoxical sleep, the possibility that either a stimulating or recording electrode has moved in the brain must be considered. If an electrode moved, then one would expect responses to change whether it be a stimulating or a recording electrode, although predicted changes with motion might be apparent only at certain 


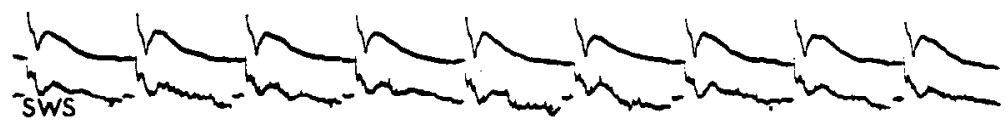

3 MIN
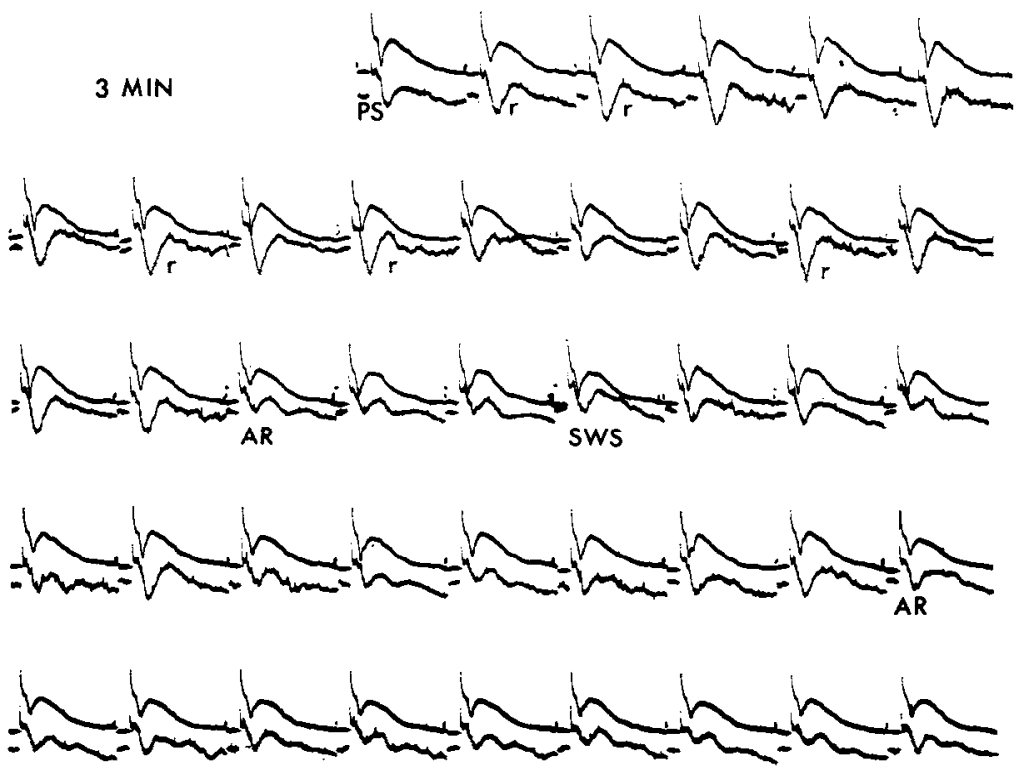

$\longrightarrow$

Frg. 3. Stimulating clectrode in fascia dentata and dorsal subiculum. Top recording from $\mathrm{CA}_{3}$ (bipolar), bottom recording from presubiculum (bipolar). A response is elicited every $5 \mathrm{sec}$ and photographed. All responses are shown except for the intervals noted. Stimulation 1.0 mamp, $1.0 \mathrm{msec}$. Calibration $50 \mathrm{msec}$ and $200 \mu \mathrm{v}$ (for both traces). Abbreviations as in Fig. 2. The response in the top trace does not change. The response in the bottun trace increases with PS.

current strengths. No such pattern was found. Often only one response from a given stimulus site would change (Figs. 2 and 3 ). If stimulation by electrode $A$ elicited a response in electrode $B$ which changed with paradoxical sleep, often the response in A from stimulation of B would not change with paradoxical sleep at any current strength. It is conceivable that some complicated motion was occurring to all electrodes simultaneously; however, single units have been recorded from some of these areas in rats during paradoxical sleep (3), (and it has been done recently in this laboratory also) without change in the size of the unit. I conclude brain motion does not occur.

The electrical impedance of the subiculum and presubiculum (collectively called the subicular area) is known to increase up to $25 \%$ during para- 
doxical sleep (4). I have also found smaller increases (about 5\%) in the entorhinal cortex during paradoxical sleep (unpublished), but no changes in hippocampus or fascia dentata. There are no impedance changes in any of these structures during arousal. Since constant-current stimuli were used, and it is the voltage gradient in tissue that stimulates, the question immediately arises as to whether increases in responses from stimulation of subiculum were due only to the increase in electrical impedance of the tissue. It seems unlikely that this is the entire explanation, since most other responses from stimulation of these areas did not increase and the amplitude of one of the responses increased two, three, and five times at different stimulating currents. This increased impedance in the subicular area, however, is clearly a contribution to increased responses from stimulation of this area.

The pathway from hippocampus to entorhinal cortex, presubiculum, and subiculum is a controversial one. Votaw described fibers in this direction (7), and a motor response mediated in this direction (6); and Adey, Sunderland, and Dunlop (1) also described responses in this direction. However, this direction of transfer, which is one affected in paradoxical sleep, often seems to be ignored.

At present, there is no clear evidence for what the mechanism of these changes might be. Macroelectrode responses in many other parts of the brain change during different states of consciousness, some increasing and some decreasing (2). In many other parts of the brain, responses which decrease with paradoxical sleep often decrease with arousal.

\section{References}

1. Adey, W. R., S. Sunderland, and C. W. Dunlop. 1957. The entorhinal area: electrophysiological studies of its interrelation with rhinencephalic structures and the brainstem. Electroencepholog. Clin. Neurophysiol. 9: 309-324.

2. Jovvet, M. 1967. Neurophysiology of the states of sleep. Physiol. Rev. 47: 117177.

3. Mink, W. D., P. J. Best, and J. Otds. 1967. Neurons in paradoxical sleep and motivated behavior. Science 158 : 1335-1337.

4. RANCK, J. B., JR. 1966. Electrical impedance in the subicular area of rats during paradoxical sleep. Exptl. Neurol. $16: 416-437$.

5. Reivich, M., G. Isaacs, E. Evarts, and S. KetY. 1968. The effect of slow wave sleep and REM sleep on regional cerebral blood flow in cats. J. Neurochem. 15: 301-306.

6. Votaw, C. L. 1959. Certain functional and anatomical relations of the cornu Ammon's of the macaque monkey. I. Functional relations. J. Comp. Neurol. 112: 353-382.

7. Votaw, C. L. 1960. Certain functional and anatomical relations of the cornu Ammon's of the macaque monkey. II. Anatomical relations. J. Comp. Neurol. 114 : 283-293. 